\title{
Effect of intervertebral disc disease on scoliosis in the lumbar spine
}

\author{
Gustaw Wojcik ${ }^{1,3 *}$, Jolanta Piskorz ${ }^{2}$, Joanna IlzeckA ${ }^{4}$, Wlodzimierz Bulikowski $^{1}$ \\ ${ }^{1}$ Department of Rehabilitation, Physiotherapy and Balneotherapy of Medical University in Lublin, Chodzki 6, 20-093 Lublin, Poland \\ ${ }^{2}$ Department of Anesthesiology and Intensive Care, Zofia Zamoyska of Tarnowski Hospital in Tarnobrzeg, Poland \\ ${ }^{3}$ Department of Diagnostic Imaging - Provincial Hospital in Staszow, Poland \\ ${ }^{4}$ Department of Rehabilitation, Physiotherapy and Balneotherapy, Independent Laboratory of Neurological Rehabilitation of Medical \\ University in Lublin, Poland
}

\begin{tabular}{l}
\hline ARTICLE INFO \\
\hline Received 2014 \\
Accepted 2014 \\
\hline
\end{tabular}

\section{Keywords:}

displacement of the

intervertebral disc,

lumbar curvature of the spine, CT.

\begin{abstract}
Lumbar intervertebral discopathy is a common clinical problem and a significant cause of low back pain. Usually it is associated with overload and damage to the intervertebral disc, which directly relates to the instability of the motion segment. Renal normal anatomy between tissues within the spinal canal is a cause of the reflex curvature of the spine, the goal is to increase the volume of the spinal channel and reduce the compression of the nerve tissue. Knee-jerk reaction to pain is an non physiological change of the setting of the spine in the frontal plane.

The aim of the study was to establish the relationship of migration of the intervertebral disc with reflex scoliosis in the lumbar region in patients with lumbar disc herniation.

Material and methods. The studies involved 78 patients aged $18-55$ years with damaged intervertebral disc with annulus fibrosus of the bulge in the posterior-lateral direction. The study used multi-row spiral CT scanner.

Results. In the patients, reflex lateral curvature was observed. The angle of scoliosis was measured with Cobb method; it did not exceed 10 degrees. It was also established that spontaneously the shallowing of lumbar lordosis and vertical alignment of the sacrum occured.

Conclusion. The study shows that in most patients diagnosed with lateral migration of the intervertebral disc there develops a slight lateral curvature of the spine, which is a reflex reaction of axial musculoskeletal to discogenic pain.
\end{abstract}

\section{INTRODUCTION}

Pains in lower back are an increasingly common complaint. Until recently, it was thought that they apply only to older people but reports in recent years testify to the fact that this problem affects younger and younger people including children [5].

Damage to the intervertebral disc causes structural abnormalities associated with a reduction of its height, and engraves the annulus outside the edge of the adjacent vertebral bodies. This is the direct cause of the instability of the motion segment. Convexities of intervertebral disc directed into the spinal canal or intervertebral holes disrupt

Corresponding author

e-mail: gustaww@tlen.pl

tel. 508247049 the anatomical conditions there between the posterior vertebral elements and the front part of the dural sac.

Lumbar intervertebral disc is a common clinical problem and a significant cause of low back pain. Symptoms of a hernia are usually characteristic but appear in varying degrees in individual patients [6].

Convexities of intervertebral disc into one of the holes cause the intervertebral nerve root compression and swelling. The abolition of the reserve volume of intervertebral canal and production of nerve root compression causes the body's immune response involving the knee-jerk skew of the spine in the opposite direction. The lumbar muscles on one side are reduced by prolonged tension, whereas the opposite side comes to the stretch. The result is a lack of proper setting of the spine, the correct shape and adequate mobility, but such setting of spinal reflex brings certain advantages, it 
results in broadening of intervertebral space and increase of the volume of the spinal channel in which previously there was a conflict tissue. Reflex back pain responses caused by nerve root compression rely on non-physiological change of its settings in the frontal plane.

Position of the spine in response to the pain does not generally exceed 10 degrees according to the Cobb test method. Scoliosis in adults with skeletal maturity is defined as the deformation of the spine with scoliosis Cobb angle above 10 degrees. Therefore reactive curvature caused by pain root, which takes place exclusively in the frontal plane of scoliosis curvatures that run on many levels must be separated [11].

If left untreated, the side curvatures lead to the fixation of asymmetric body burden and degenerative spinal deformity, which can grow up to the end of life. The rate of progression of scoliosis is 0.5-1.0 degrees a year [11].

According to Cobb, the angle of scoliosis of less than 5 degrees is present in $42.8 \%$ of the healthy population, and the angle of curvature of less than 10 degrees refers to $17.6 \%$ of the healthy population.

\section{AIM}

The aim of the study was to establish the relationship of migration of the intervertebral disc with reflex scoliosis in the lumbar region in patients with lumbar disc herniation.

\section{MATERIAL AND METHODS}

The studies involved 83 patients: 41 women and 42 men. The age range of respondents was between 18 and 55 years of age. The average age for women was 45.9 years and for men 40.6 years.

CT scan was performed in patients with pain due to lumbar-sacral spine. All patients gave their consent to participate in the above diagnostic test.

The study concerned patients with pain in the lower lumbar spine where convexities of nucleus pulposus had a narrow base and migrated towards one intervertebral opening. Another factor, which qualified for the study, was the occurrence of primary lateral curve of the spine without compensating secondary side bends, which are a manifestation of efforts to compensate the mechanical disorders of the spine axis, and without rotation of the vertebrae. The study also included patients who never underwent treatment of discectomy or other operations in the spine.

The algorithm excluding patients from the study included the existence of spinal canal stenosis associated with pathological proliferation of bone tissue in the form of osteophytes on the posterior surface of the vertebral joints and hypertrophy outgrowths reducing side intratrabecular recesses of the spinal canal. Studies also excluded patients with malformations of the half characteristics of extremely situated vertebrae and fractures of the lumbar spine as well as patients with structural scoliosis. Studies also excluded patients diagnosed with CT calcifications within longitudinal ligament of the front and rear.

The study was performed with 16-row CT scanner Philips Brilliance in $1 \mathrm{~mm}$ layers. Analysis of lateral curvature of the lumbar spine was performed by diagnostic station
Carestream Health using an algorithm for determining the Cobb angle.

Cobb angle was determined by lines extending parallel to the vertebral endplates of the greatest deviation from the axis side of the spine. The top line ran through the circle of proximal lamina lying above the curvature, while the lower through the lamina distal farthest circle arc curvature. Then the lines perpendicular to the previously established parallel lines were determined and the angle of intersection of the perpendicular angle defined curvature of the spine - Cobb angle.

Cobb method - a diagnostic method for calculating the degree of curvature of the spine. It consists in plotting the straight lines along the top surface of the upper limit of the curvature circle and the bottom surface of the lower limit circle, then drawing a straight perpendicular to these lines. These lines intersecting the side of the bending angle indicate the degree of bending of the spine. For practical reasons, not correct angle is taken into account, but the complementary angle (upper or lower). The value of this angle increases with increasing deformation. If it exceeds 10 degrees, a patient is diagnosed with scoliosis.

In the examined patients, there were also performed measurements of cross-sectional diameter of both lumbar muscles on the height of L4 in order to determine the relationship of linear muscle direction of the lateral curvature of the spine in the lumbar region.

The study was conducted in 2009-2011 after a positive opinion of the Bioethics Committee at the Medical University of Lublin. The opinion of the Bioethics Committee : KE-0254/130/2008 of 03.07.2008.

\section{RESULTS}

The calculated average values of the angle of curvature of the spine by Cobb method indicate that in women they amounted to 5.40 degrees and in men to 5.55 degrees. The average size of disc herniation was $5.63 \mathrm{~mm}$ for women and $6.39 \mathrm{~mm}$ for men.

Table 1. Parameter values associating curvature of the spine broken by gender

\begin{tabular}{|l|c|c|}
\hline & Women & Men \\
\hline Number of examined $(n)$ & 41 & 42 \\
\hline Side of curvature convexity (R/L) & $20 / 21$ & $20 / 22$ \\
\hline Average age & $45.9(9.49$ SD) & $40.6(11.50)$ \\
\hline Average value of the Cobb angle (X0) & $5.28(2.21$ SD) & $5.58(2.35$ SD) \\
\hline Size of disc convexity (mm) & $5.62(1.04 S D)$ & $6.46(1.35$ SD) \\
\hline
\end{tabular}

Based on the survey, there was no trend towards more frequent protrusion of the spinal curvature in either direction for either sex. Given that the majority of the Polish population is right-handed - this had no influence on the direction of curvature of the spine in the frontal plane.

Average size of intervertebral disc conversities was larger in the case of men, and the difference in the size reached $13 \%$. Measurements of the average angles of curvature by the Cobb method were almost identical for both sexes with a slightly greater curvature in men (5.58 degrees). 
The analysis of studies showed that in the case of lateral lumbar disc herniation it usually comes to setting the lumbar spine reflex arc bending in the opposite direction to the occurrence of hernia.

Spontaneously it comes to shallowing of lumbar lordosis and vertical alignment of the sacrum. The pelvis is inclined back.

Table 2. Average values of the surface area of both lumbar muscles measured at a height of L4 vertebra broken by gender

\begin{tabular}{|c|c|c|c|c|c|c|c|c|}
\hline & \multicolumn{4}{|c|}{ Women } & \multicolumn{4}{|c|}{ Men } \\
\hline & 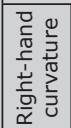 & जे & 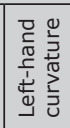 & î & 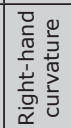 & ตे & 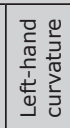 & जे \\
\hline $\begin{array}{l}\text { Field area of the right } \\
\text { lumbar muscle }(\mathrm{mm})\end{array}$ & 10.09 & 1.44 & 10.41 & 1.58 & 15.52 & 3.14 & 16.75 & 2.47 \\
\hline $\begin{array}{l}\text { Field area of the right } \\
\text { lumbar muscle }(\mathrm{mm})\end{array}$ & 10.28 & 1.46 & 9.78 & 1.98 & 15.89 & 3.28 & 15.31 & 2.45 \\
\hline
\end{tabular}

Average surface area measurements made on the lumbar muscle at L4 showed that for both sexes on curvature of the convex side of the lumbar section, muscles are always of smaller volume than on the opposite side.

\section{DISCUSSION}

Diagnostic imaging studies are an integral part of the clinical trial in patients with back pain. The primary role of imaging is to identify the cause of the problem, on the basis of which the effective treatment is included [8].

Degenerative changes extended over time are mild, which may hinder the initial diagnosis solely on the basis of physical examination. Severe radiographic image is not proportional to the intensity of pain in the patient. It is a very poor correlation between the results of prolapsed disk imaging and clinical course of disc disease [8].

Neuromuscular scoliosis is usually formed at a young age, but may also develop in adulthood. Its characteristic feature is a rapidly progressive curvature of the spine [2].

Structural changes in the soft tissue associated with a herniated nucleus pulposus center toward the rear and cause a compensatory change in the axis of the spine, by eliminating the physiological lumbar lordosis, and result in an increase in channel capacity and create more favorable conditions for the contained nerve tissue. Compensatory position of the spine is also the case of a herniated intervertebral side of the hole, with the exception that due to ejection of the disc there is abnormal lateral curvature of the spine to form a curvature in the frontal plane.

In the case of lateral curvature of the spine reaching 10 degrees by the Cobb method of measurement, there is a significant shortness of lumbar lordosis with a more vertical rotation of the sacrum. The formation of non-physiological curvature may increase the compressive forces and as a result - lead to progressive degeneration of intervertebral discs [12].

The rapid increase in body height, especially during puberty can cause spinal deformity. Abnormal development of paraspinal muscles can cause scoliosis, but in the small curvature of the side it is not the change of length and volume of the muscle that cause deformation, but rather transfer of the intervertebral disc $[3,9]$.
In most patients with severe scoliosis one can observe increased degenerative changes in intervertebral channels within the concave side of the curvature causing compression of nerve roots and triggering pain distribution to the lower limb on the same side [4].

In degenerative scoliosis, nerve root compression is the result of irritation of the periosteum and growth of degenerative changes in the intervertebral channels, at the same time producing a severe orifice on the concave side of the curvature. This curvature coincides with vertebral rotation along the long axis of the spine, and the curvature of the spine reflex bending is formed as a defensive reaction to pain root and runs only in the frontal plane. Degenerative scoliosis usually meets with the Cobb angle greater than 15 degrees.

Scoliosis is closely linked to degeneration of discs, and unbalanced load causes progressive degeneration of the intervertebral disc [1]. Its convexity at L5/S1 can be an important predictor of curvature of the spine [10].

Hemilaminoplastics allows a reduction of the Cobb angle, which results in the restoration of a more favorable relationship of the lumbar spine. This technique gives good clinical and radiological results [14].

After adjusting for surgical lumbar disc herniation, alleviated irritation of the nerve roots occurs. The Cobb angle measurements state clinical and radiological regression of pathological settings of the spine. After a few months of the treatment after surgery, there comes the widening of the intervertebral holes on the concave side of the curvature and the reduction of the Cobb angle [7].

After surgical decompression, pain and numbness in the extremities subside in patients. The spontaneous correction of scoliosis also follows [16].

The direction of curvature of the spine is strongly associated with the direction of incidence of hernia, and early decompression may provide a greater possibility of spontaneous correction of scoliosis $[13,15]$.

\section{CONCLUSION}

The study shows that most patients diagnosed with lateral migration of the intervertebral disc undergo a slight lateral curvature of the spine, which is a reaction to the reflex discogenic pain.

\section{REFERENCES}

1. Buttermann GR., Beaubien BP. In vitro disc pressure profiles below scoliosis fusion constructs. Spine. 33(20): 2134-42, 2008

2. De Baat P., Van Biezen FC., De Baat C. Scoliosis: review of types, a etiology, diagnostics, and treatment 2. Ned Tijdschr Tandheelkd. 119(11): 531-5, 2012

3. Duart Clemente J., Llombart Blanco R., Beguiristain Gurpide JL. Morphological changes in scoliosis during growth. Study in the human spine. Rev Esp Cir Ortop Traumatol. 56(6): 432-8, 2012.

4. Fu KM. et al. Prevalence, severity, and impact of foraminal and canal stenosis among adults with degenerative scoliosis. Neurosurgery. 69(6): 1181-7, 2011.

5. Furnes O., Bøe A., Sudmann E. Lumbar intervertebral disk prolapse in adolescents. Tidsskr Nor Laegeforen. 116 (25): 2993-5, 2001.

6. Huang SR., Shi YY., Zhan HS. Diagnostic ideas and programs of lumbar intervertebral disc herniation. China J Orthop Traumatol. 25(2): 147-51, 2012. 
7. Matsui H., Ohmori K., Kanamori M. Significance of sciatic scoliotic list in operated patients with lumbar disc herniation. Spine. 23(3): 338-42, 1998.

8. Maus T. Imaging the back pain patient. Phys Med Rehabil Clin N Am. 21(4): 725-66, 2010.

9. Murakami N. et al. Scoliosis In Prader-Willi syndrome: effect of growth hormone therapy and value of paravertebral muscle volume by CT In predicting scoliosis progression. Am J Med Genet A. 158A(7): 1628-32, 2012.

10. Pritchett JW., Bortel DT. Degenerative symptomatic lumbar scoliosis. Spine. 18(6): 700-3, 1993.

11. Quante M. et al. Die operative Behandlung der adulten Scoliose. Osteoporose und Versagen des Anschlusssegments als besondere
Herausforderung bei der operative Versorgung. Der Orthopäde. 38(2): 159-69, 2009.

12. Rajnics P. et al. The importance of spinopelvic parameters in patients with lumbar disc lesions. Int Orthop. 26(2): 104-8, 2002.

13. Suk KS., Lee HM., Moon SH. Lumbosacral scoliotic list by lumbar disc herniation. Spine. 26(6): 667-71, 2001.

14. Xinyu L., Yanping Z., Jianmin L. Hemilaminoplasty for the treatment of lumbar disc herniation. Int Orthop. 33(5): 1323-7, 2009.

15. Zhu Z. et al. Scoliotic posture as the initial symptom in adolescents with lumbar disc herniation: its curve pattern and natural history after lumbar discectomy. BMC Musculosceletal Disord. 12: 216, 2011.

16. Zhu ZZ. et al. Clinical evaluation of adolescent lumbar disc herniation misdiagnosed as idiopathic scoliosis. Chin J Surg. 46(14): 1058-61, 2008. 